Documentation et bibliothèques

DOCUMENTATION BIBLIOTHEQUES

\title{
Le prêt entre bibliothèques : un outil à repenser
}

\section{Onil Dupuis et John Allan Hobbins}

Volume 25, numéro 1, mars 1979

URI : https://id.erudit.org/iderudit/1054366ar

DOI : https://doi.org/10.7202/1054366ar

Aller au sommaire du numéro

Éditeur(s)

Association pour l'avancement des sciences et des techniques de la documentation (ASTED)

\section{ISSN}

0315-2340 (imprimé)

2291-8949 (numérique)

Découvrir la revue

\section{Citer cet article}

Dupuis, O. \& Hobbins, J. A. (1979). Le prêt entre bibliothèques : un outil à repenser. Documentation et bibliothèques, 25(1), 3-10.

https://doi.org/10.7202/1054366ar

\section{Résumé de l'article}

Un groupe de travail du Sous-comité des bibliothèques de la Conférence des recteurs et des principaux des universités du Québec a étudié les problèmes nouveaux du prêt entre bibliothèques. Une étude rapide des principaux délais des opérations de prêt et d'emprunt entre bibliothèques et de leurs causes précède l'analyse de deux facteurs ou moyens pouvant contribuer à diminuer ces délais.
Tous droits réservés ( $)$ Association pour l'avancement des sciences et des techniques de la documentation (ASTED), 1979
Ce document est protégé par la loi sur le droit d'auteur. L'utilisation des services d'Érudit (y compris la reproduction) est assujettie à sa politique d'utilisation que vous pouvez consulter en ligne.

https://apropos.erudit.org/fr/usagers/politique-dutilisation/ 


\section{Le prêt entre bibliothèques: un outil à repenser}

\section{Onil Dupuis}

Conférence des recteurs et des

principaux des universités du Québec

Montréal

\section{John Allan Hobbins}

Bibliothèque McLennan

Université McGill

Montréal

Un groupe de travail du Sous-comité des bibliothèques de la Conférence des recteurs et des principaux des universités du Québec a étudié les problèmes nouveaux du prêt entre bibliothèques. Une étude rapide des principaux délais des opérations de prêt et d'emprunt entre bibliothèques et de leurs causes précède l'analyse de deux facteurs ou moyens pouvant contribuer à diminuer ces délais.

A task force of the library Sub-committee of the "Conférence des recteurs et des principaux des universités du Québec" has made a study of the new problems involved in interlibrary loans. A rapid review of the main delays implied in these loans, and of the causes thereof, precedes the analysis of two factors or means which could help reduce these delays.

Un grupo de trabajo del Subcomité de las bibliotecas de la Conferencia de los rectores y principales de las universidades de Quebec estudió los nuevos problemas del préstamo interbibliotecario. Un breve estudio de las principales tardanzas en las operaciones de préstamo interbibliotecario y de sus causas precede el análisis de dos factores o medios que podrian contribuir a disminuir los retrasos.

Le prêt entre bibliothèques constitue un excellent moyen de compléter des collections déficientes, moyen indispensable à tout effort sérieux de rationalisation du développement des bibliothèques. Il correspond à la poursuite de deux principaux objectifs:

1) utilisation plus rationnelle et plus économique des ressources des différentes bibliothèques;

2) possibilité pour chaque bibliothèque d'offrir à ses usagers des services d'information de plus haute qualité. 
Depuis quelques années cependant, des abus dans la façon d'utiliser ce service et des restrictions budgétaires causées par une situation économique difficile ont amené plusieurs grandes bibliothèques à imposer des mesures d'austérité qui, généralisées, risquent de constituer une entrave très sérieuse aux différents efforts de rationalisation et de coopération entre bibliothèques entrepris sous la pression même de ces contraintes économiques.

La décision de facturer les transactions du prêt entre bibliothèques constitue, de l'aveu même des bibliothèques qui ont adopté cette politique, un palliatif et non une solution véritable aux difficultés réelles causées par une utilisation accrue (et non pas nécessairement abusive) de ce privilège.

Soucieuses des répercussions immédiates d'une éventuelle décision similaire sur l'ensemble des bibliothèques québécoises, les bibliothèques universitaires du Québec ont préféré entreprendre l'étude de solutions permettant d'améliorer l'efficacité, la rapidité et la rentabilité du prêt entre bibliothèques qui ne restreignent pas l'accessibilité aux documents.

C'est dans ce but que le Sous-comité des bibliothèques de la Conférence des recteurs et des principaux des universités du Québec créait, au mois d'août 1977, un groupe de travail chargé d'étudier les moyens les plus appropriés d'améliorer l'efficacité, la rapidité et la rentabilité des services de prêt entre bibliothèques, sans oublier d'identifier les difficultés éventuelles posées par l'application de ces moyens.

La création de ce groupe de travail s'inscrivait dans le cadre des efforts entrepris par le Sous-comité afin d'améliorer la qualité des services offerts aux usagers des bibliothèques universitaires du Québec, notamment en leur facilitant l'accès aux ressources documentaires de l'ensemble des bibliothèques universitaires, soit directement (réciprocité du privilège d'emprunt direct), soit par l'intermédiaire de leur bibliothèque (emprunt entre bibliothèques).

Le présent article constitue davantage un résumé d'une partie des travaux du groupe de travail ${ }^{1}$ qu'un rapport exhaustif sur les implications des différents facteurs pouvant influencer l'efficacité, la rapidité ou la rentabilité des opérations de prêts entre bibliothèques.

Après un rapide survol des principaux délais des opérations de prêt et d'emprunt entre bibliothèques et de leurs causes, nous étudierons deux des facteurs ou des moyens pouvant contribuer à diminuer ces délais.

\section{Les délais de prêt et d'emprunt}

Dans le but d'identifier les principales causes des délais de prêt et d'emprunt entre bibliothèques, le groupe de travail s'est d'abord attaché à réaliser une étude détaillée des différentes étapes du

1. Le groupe de travail était composé de Marie-Andrée Beaupré, Onil Dupuis, John Allan Hobbins, Clément Tremblay et Derek Robertson. 
cheminement ou des différentes opérations relatives aux prêts ou aux emprunts de documents et de photocopies et à identifier les délais minimum et moyen relatifs à chaque opération.

En ce qui concerne les documents, la bibliothèque emprunteuse doit d'abord localiser le document désiré dans un catalogue collectif. Les principaux catalogues collectifs disponibles sont ceux de l'Université du Québec, BADADUQ, de la Bibliothèque nationale du Canada (BNC), de l'Institut canadien pour l'information scientifique et technique (ICIST) et TELECAT/UNICAT. Malgré les recommandations de différents organismes nationaux et provinciaux, il n'existe encore aucun catalogue collectif des ressources documentaires québécoises (ni des autres provinces). En conséquence, la plupart des demandes de localisation sont adressées à la Bibliothèque nationale du Canada.

Dans le cas des photocopies d'articles de périodiques, il existe plusieurs outils de localisation mis à la disposition des bibliothèques, outils qui, la plupart du temps, permettent de localiser rapidement le périodique désiré, sans avoir à recourir à un organisme extérieur. La bibliothèque emprunteuse peut ainsi adresser directement sa demande à la bibliothèque prêteuse, ce qui réduit considérablement le délai d'obtention du document.

Dans les deux cas cependant, des délais supplémentaires considérables peuvent s'ajouter lorsque la bibliothèque qui reçoit la demande n'est pas en mesure de fournir le document demandé parce qu'il est «déjà prêté», qu'il «ne circule pas», ou qu'il est tout simplement "perdu». La bibliothèque emprunteuse doit parfois s'adresser ainsi à deux ou trois bibliothèques avant de compléter une transaction.

Selon les données obtenues à la suite d'une enquête sommaire effectuée auprès des quatre institutions représentées au groupe de travail, le délai moyen d'obtention d'une localisation de la Bibliothèque nationale du Canada est de trois jours. Cette donnée confirme celles obtenues de la Bibliothèque nationale du Canada à l'effet qu'environ $76 \%$ des demandes qui lui sont adressées (par télex) reçoivent une réponse dans les trois jours qui suivent et $99 \%$ des demandes dans les cinq jours qui suivent ${ }^{2}$. Mentionnons cependant qu'il s'agit là d'une amélioration récente de la rapidité du service de localisation offert par la Bibliothèque nationale du Canada. Ainsi, en 1976, une enquête effectuée par l'Université de Waterloo indiquait un délai moyen de localisation de 8.6 jours $^{3}$.

Le délai moyen d'obtention d'une photocopie d'une bibliothèque canadienne est de douze jours. Le délai moyen d'obtention d'un prêt de documents est de quinze jours. Les résultats obtenus en 1976 lors de l'enquête effectuée par l'Université de Waterloo indiquaient des délais de l'ordre de 11.5 jours et de 23.24 jours respectivement et confirment les résultats de l'enquête sommaire effectuée par le groupe de travail.

2. Ramma Kamra, Interlibrary Loans as it Touches the National Library of Canada, prepared for the CASLIS Toronto Chapter's meeting on «Interloan: form and essence», Toronto, October 5, 1977, p. 5.

3. Alexander Slade and Merylyn Somerville, Interlibrary Loan Survey, Waterloo, University of Waterloo, 1976, p. 1. 
Il est à souligner que les délais rapportés plus haut ne concernent que les demandes expédiées par télex. Par ailleurs, le délai moyen de transport des documents obtenus d'une bibliothèque membre du réseau PEBUQUILL / IUTS est d'un jour, alors que le délai moyen de réception des documents obtenus de bibliothèques qui ne sont pas reliées au réseau est de six jours supplémentaires.

\section{Etude de differents facteurs pouvant affecter les délais de prêt et d'emprunt entre bibliothèques}

\section{Catalogue collectif des collections des bibliothèques universitaires du Québec}

Il est certain que l'absence de catalogue collectif des collections des bibliothèques universitaires (et non universitaires) du Québec et des autres provinces constitue un facteur important pouvant contribuer à l'augmentation considérable des délais de prêt et d'emprunt entre bibliothèques. D'une part, toutes les demandes de localisation doivent être adressées à la Bibliothèque nationale du Canada, ajoutant ainsi un délai moyen de deux à trois jours ${ }^{4}$. D'autre part, il est difficile d'assurer une bonne répartition des demandes d'emprunt entre les différentes bibliothèques, ce qui contribue de façon significative à augmenter le volume de transactions déjà élevé des institutions les plus importantes.

Ce sont d'ailleurs ces mêmes raisons qui ont amené le Conseil d'administration de l'Association des universités et collèges du Canada, lors de sa réunion du 10 août 1976, à recommander:

76.2.6 «Que les recteurs des établissements membres de l'AUCC assurent que le service de prêts de leur bibliothèque fasse des démarches pour repérer le document demandé auprès des services les plus rapprochés; et si le document n'est pas disponible dans la région, que la mention «il est impossible de repérer le document visé dans la région» soit inscrite sur la formule de demande et signée par le bibliothécaire compétent avant de transmettre celle-ci à un service plus éloigné; ”

76.2.10 «Que les recteurs des établissements membres de l'AUCC assurent que leur établissement tienne un répertoire complet de ses périodiques et le fasse parvenir à tous les établissements voisins et les bibliothèques nationales».

Par ailleurs, le Sous-comité des bibliothèques, dans le but de donner suite à cette recommandation, créait, lors de sa $65^{\mathrm{e}}$ réunion tenue le 13 octobre 1976, un groupe de travail chargé d'étudier les méthodes les plus appropriées de contrôle bibliographique des périodiques.

De plus, le 18 novembre 1977, le Sous-comité a tenu une réunion des directeurs des bibliothèques des établissements universitaires du Québec dans le but d'étudier la possibilité de l'établissement d'une

4. Ramma Kamra, Interlibrary Loans..., p. 5. 
liste collective automatisée des périodiques des bibliothèques universitaires du Québec.

Le groupe de travail considère essentiel à l'amélioration de l'efficacité et de la rapidité du prêt entre bibliothèques l'établissement d'un catalogue collectif des périodiques des bibliothèques universitaires du Québec.

Cependant, informé des travaux en cours relativement au projet de conversion rétrospective des fichiers des usagers de TELECAT/ UNICAT, et compte tenu de la disponibilité actuelle du catalogue collectif des collections des bibliothèques de l'Université du Québec, via BADADUQ, le groupe de travail n'a pas voulu pousser plus loin cette étude de l'impact éventuel d'un catalogue collectif des collections des bibliothèques universitaires du Québec.

\section{Utilisation de TELECAT/UNICAT pour le prêt entre bibliotheques}

Parmi les moyens susceptibles de réduire à la fois les délais et les coûts des services de prêts entre bibliothèques, l'utilisation des systèmes automatisés reliant les institutions universitaires entre elles constitue celui qui présente le plus d'intérêt. Le système BADADUQ qui relie les membres du réseau de l'Université du Québec est déjà utilisé à cette fin depuis sa création. Cependant, aucune étude n'avait encore été entreprise quant aux possibilités d'utilisation du système TELECAT/UNICAT, qui compte parmi ses usagers québécois toutes les bibliothèques universitaires du Québec (à l'exception de l'Université du Québec), la Bibliothèque nationale du Québec, la bibliothèque administrative et la bibliothèque de l'Hydro-Québec. A ceux-ci s'ajoutent dix usagers ontariens dont sept (bientôt huit) bibliothèques universitaires, deux bibliothèques publiques et une bibliothèque gouvernementale.

En janvier 1978, une enquête sommaire permit d'évaluer l'efficacité du système TELECAT/UNICAT en comparaison avec les services de la Bibliothèque nationale du Canada en ce qui concerne la localisation des documents ${ }^{5}$. Cette enquête visait à déterminer les types de documents pour lesquels le système TELECAT/UNICAT pouvait constituer une alternative valable aux services de la BNC.

Les avantages et les inconvénients de l'utilisation du catalogue collectif canadien sont connus. Les collections de la plupart des grandes bibliothèques canadiennes y sont répertoriées. Par contre, les délais de classement des fiches dans ce catalogue peuvent parfois être assez importants. Le délai moyen entre le moment où un document est catalogué par une institution et le moment de son insertion au catalogue collectif est d'environ un an. Le délai moyen de réponse de la BNC aux demandes de localisation est de deux à trois jours. Enfin, les documents y sont répertoriés uniquement par l'entrée principale.

5. John A. Hobbins, The Results of a Survey to Investigate an Alternative to the National Library as a Source of Canadian Locations, Montreal, McGill University, McLennan Library, 1978. 
Par ailleurs, l'utilisation du système TELECAT/UNICAT comporte également des avantages et des inconvénients. La principale caractéristique du système est, bien sûr, l'actualité de son fichier collectif: les dossiers y sont versés dès leur création.

Tous les dossiers sont accessibles directement (ou dans un délai maximum de 24 heures) soit par titre, soit par no ISBN ou encore par numéro de la fiche de la Library of Congress. Enfin, la plupart des institutions membres du système sont reliées entre elles grâce au service de messageries PEBUQUILL / IUTS.

Par contre, le fichier collectif contient peu de documents catalogués avant 1973, à l'exception de la collection de la bibliothèque de l'Université de Toronto.

L'échantillonnage utilisé pour les fins de cette enquête fut limité à cent titres publiés entre 1973 et 1976 dans dix-sept pays et dans les langues suivantes: anglais, français, allemand et italien. Les sujets des documents choisis se rapportaient exclusivement au secteur des sciences humaines et sociales.

\section{Echantillonnage de l'enquête}

1. L'échantillonnage comprend 100 documents

2. Clés d'accès:

ISBN

Fiche du congrès

Titre seulement

3. Langues:

Anglais

Français

Allemand

Italien

4. Date de publication: 1973

1974

5. Lieu de publication: Allemagne

Australie

Autriche

Belgique

Canada

Etats-Unis

France

Grande-Bretagne

Grèce

Hongrie

Italie

Kenya

Malaisie

Pays-Bas

Suède

Suisse 
Les documents du secteur des sciences humaines et sociales publiés entre 1973 et 1976 constituent, pour les établissements universitaires francophones, $23.46 \%$ du total des emprunts de documents de ce secteur et $18.89 \%$ du total des emprunts ou des prêts. Chez les institutions universitaires anglophones, ce pourcentage est respectivement de $16.49 \%$ et de $7.45 \%^{6}$.

Les réponses obtenues de l'une et l'autre source ont été comparées entre elles d'abord selon le nombre total de localisations ainsi obtenues et, ensuite, en fonction d'une pondération accordée à chacune des localisations obtenues. Pour les fins de cette enquête, les critères de choix du lieu éventuel de l'emprunt ont été établis comme suit:

1. Montréal.

2. Bibliothèques membres du réseau PEBUQUILL / IUTS.

3. Toutes les bibliothèques canadiennes, à l'exception de celles qui facturent les prêts entre bibliothèques.

4. Bibliothèques qui facturent les prêts entre bibliothèques.

Les résultats ainsi obtenus sont surprenants. La BNC a fourni 69 réponses, ce qui est légèrement inférieur à la moyenne nationale qui est de $78.3 \%$. Pour sa part, TELECAT/UNICAT a fourni 83 réponses. Seulement dix titres n'ont pu être localisés par l'une ou l'autre source. Tel que prévu, la BNC a identifié davantage de volumes publiés aux environs de 1973, alors que les plus récents l'ont été par TELECAT/ UNICAT. La langue et le lieu de publication ne semblent pas constituer des facteurs significatifs.

Les résultats de cette enquête sommaire permettent d'affirmer que le système TELECAT/UNICAT constitue un outil efficace afin d'améliorer la rapidité et le coût des opérations de prêt et d'emprunt entre bibliothèques, tout au moins en ce qui concerne les documents se rapportant aux sciences humaines et sociales.

L'échantillonnage restreint utilisé empêche toute extrapolation relative aux documents d'autres disciplines. Cependant, les résultats obtenus sont suffisamment encourageants pour justifier une étude plus approfondie portant sur un échantillonnage plus large. De plus, il serait intéressant d'envisager l'utilisation du système à des fins de vérification de même que d'étudier la possibilité d'utiliser le terminal pour les demandes d'emprunts.

L'utilisation d'un tel système, ajoutée aux possibilités d'utilisation du système BADADUQ, pourrait constituer un élément important dans la recherche d'une solution permettant d'améliorer l'efficacité, la rapidité et même la rentabilité des opérations de prêt entre bibliothèques.

6. Basil Stuart-Stubbs et al., Interlibrary Loan in Canada: a Report of a Survey, Vancouver, University of British Columbia Library, 1975, ix, $236 \mathrm{f}$. 
Tableau des localisations obtenues par langue et par date

\begin{tabular}{|c|c|c|c|c|c|}
\hline & FRANCE & $\begin{array}{l}\text { GRANDE- } \\
\text { BRETAGNE }\end{array}$ & ALLEMAGNE & ITALIE & TOTAL \\
\hline $\begin{array}{l}1973 \\
\text { Total } \\
\text { TELECAT/UNICAT } \\
\text { BNC }\end{array}$ & $\begin{array}{ll}21 & \\
17 & (80.95 \%) \\
21 & (100 \%)\end{array}$ & $\begin{array}{ll}3 & \\
3 & (100 \%) \\
3 & (100 \%) \\
\end{array}$ & $\begin{array}{l}5 \\
3(60 \%) \\
4(80 \%)\end{array}$ & - & $\begin{array}{ll}29 & \\
23 & (79.31 \%) \\
28 & (96.55 \%)\end{array}$ \\
\hline $\begin{array}{l}1974 \\
\text { Total } \\
\text { TELECAT/UNICAT } \\
\text { BNC }\end{array}$ & $\begin{array}{l}17 \\
15(88.24 \%) \\
12(70.59 \%) \\
\end{array}$ & $\begin{array}{ll}1 & \\
1 & (100 \%) \\
1 & (100 \%) \\
\end{array}$ & $\begin{array}{l}4 \\
4(100 \%) \\
4(100 \%)\end{array}$ & $\begin{array}{ll}4 & \\
0 & (0 \%) \\
0 & (0 \%) \\
\end{array}$ & $\begin{array}{ll}26 & \\
20 & (76.92 \%) \\
17 & (65.38 \%) \\
\end{array}$ \\
\hline $\begin{array}{l}1975 \\
\text { Total } \\
\text { TELECAT/UNICAT } \\
\text { BNC }\end{array}$ & $\begin{array}{l}17 \\
16(94.12 \%) \\
15(88.24 \%)\end{array}$ & $\begin{array}{lr}2 & \\
2 & (100 \%) \\
1 & (50 \%)\end{array}$ & $\begin{array}{l}7 \\
5 \\
2(71.43 \%) \\
2(28.57 \%)\end{array}$ & 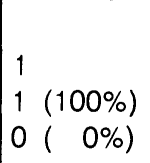 & $\begin{array}{ll}27 & \\
24 & (88.89 \%) \\
18 & (66.67 \%)\end{array}$ \\
\hline $\begin{array}{l}1976 \\
\text { Total } \\
\text { TELECAT/UNICAT } \\
\text { BNC }\end{array}$ & $\begin{aligned} 13 & \\
12 & (92.31 \%) \\
6 & (46.15 \%)\end{aligned}$ & $\begin{array}{lr}3 & \\
3 & (100 \%) \\
0 & (\quad 0 \%)\end{array}$ & $\begin{array}{lr}2 & \\
1 & (50 \%) \\
0 & (0 \%)\end{array}$ & - & $\begin{array}{l}18 \\
16(88.89 \%) \\
6(33.33 \%)\end{array}$ \\
\hline $\begin{array}{l}\text { TOTAL } \\
\text { Total } \\
\text { TELECAT/UNICAT } \\
\text { BNC }\end{array}$ & $\begin{array}{l}68 \\
60(88.24 \%) \\
54(79.41 \%)\end{array}$ & $\begin{array}{l}9 \\
9(100 \%) \\
5(62.5 \%)\end{array}$ & $\begin{array}{l}18 \\
13(72.22 \%) \\
10(55.56 \%)\end{array}$ & $\mid \begin{array}{lr}5 & \\
1 & (20 \%) \\
0 & (0 \%)\end{array}$ & $\begin{array}{r}100 \\
83 \\
69\end{array}$ \\
\hline
\end{tabular}

Conclusion

Les services de prêt entre bibliothèques constituent le moyen privilégié permettant de mettre à la disposition des usagers de chaque bibliothèque les collections de l'ensemble des bibliothèques.

Dans la perspective d'une rationalisation éventuelle des politiques de développement des collections des bibliothèques universitaires du Québec, et dans l'optique des efforts de collaboration et de coordination déjà entrepris par le Sous-comité des bibliothèques, l'amélioration de l'efficacité, de la rapidité et de la rentabilité des services de prêt entre bibliothèques constitue un élément essentiel au fonctionnement efficace du réseau des bibliothèques universitaires québécoises et, à ce titre, devrait faire l'objet de ses priorités d'études au cours des prochaines années. 